\title{
Rapid screening test of most frequent BRCA1/BRCA2 pathogenic variants in the NGS era
}

\author{
D. ZIDEKOVA ${ }^{1}$, I. WACZULIKOVA ${ }^{2}$, L. DOLESOVA ${ }^{1}$, L. VAVROVA ${ }^{1}$, O. HAMIDOVA ${ }^{1}$, R. LOHAJOVA BEHULOVA ${ }^{1,3}$, M. KONECNY $^{1,4, *}$
}

${ }^{1}$ Department of Clinical Genetics, St. Elizabeth Cancer Institute, Bratislava, Slovakia; ${ }^{2}$ Division of Biomedical Physics, Department of Nuclear Physics, Biophysics, Faculty of Mathematics, Physics and Informatics, Comenius University, Bratislava, Slovakia; ${ }^{3}$ Institute of Genetics and Molecular Medicine Viliama Izakovica, Faculty of Medicine, Slovak Medical University in Bratislava; ${ }^{4}$ GHC Genetics SK, Comenius University Science Park, Bratislava, Slovakia

*Correspondence: konecny@ghc.sk

Received May 7, 2017 / Accepted August 16, 2017

\begin{abstract}
The average risk of breast cancer in general Slovak population of women is $4-5 \%$ and the risk of ovarian cancer is $2 \%$. Probability of breast/ovarian cancer development is higher in individuals carrying a causative germline DNA variant in $B R C A 1$ or $B R C A 2$ gene responsible for hereditary breast/ovarian cancer (HBOC). Although a major proportion of inherited breast/ovarian cancers are due to the mentioned causal mutations, a number of new genes have emerged. Here we describe a rapid, multiplex and comprehensive approach for the detection of pathogenic variants in $B R C A 1$ and $B R C A 2$ genes which most frequently occur in Slovak HBOC population. Analysis comprises the combination of mutation specific methods. Fluorescent PCR amplification followed by fragment analysis for detection of insertions/deletions in exon 11 of BRCA1 gene. Second method is SNaPshot analysis for detection of the most frequent missense and ins/del variants in exons 2, 5, 13, 20 of BRCA1 and exons 11, 23 and 25 of BRCA2 gene. Altogether, we have analyzed 687 samples, 86 (12.5\%) in group 1, which fulfilled indication criteria based on the positive family/personal history. Group 2 involved 601 (87.5\%) cases, who did not meet the indication criteria and only the screening test was recommended. Using the combined approach, we have identified 47 mutated samples (6.8\%), 40 in group 1 (46.5\%) and 7 in group 2 (1.1\%). However, the presented screening test would not provide complex results of $B R C A 1 / 2$ gene analysis, it offers testing accessible to a broader spectrum of individuals under the threshold of indication for whole gene analysis. This approach may provide valuable information even in the NGS analysis era.
\end{abstract}

Key words: BRCA1, BRCA2, screening test, SNaPshot analysis, pathogenic variant

Breast cancer is one of the most common cancers and one of the leading causes of cancer-related death among women [1]. It has been estimated that $10-12 \%$ of women will develop breast cancer over the course of their life [2]. The average risk of breast cancer in general Slovak population of women is $4-5 \%$ and of ovarian cancer $2 \%$ [3].

In general, the presence of breast cancer in any first-degree female relative nearly doubles the risk for an individual and the risk gradually increases with the number of affected relatives [4]. It is estimated that approximately $5-10 \%$ of all patients with breast cancer and approximately $20 \%$ of all patients with ovarian cancer exhibit a monogenic predisposition to breast and ovarian cancer [5]. Currently, more than 25 genes have been associated with familial breast and/ or ovarian cancer, but still BRCA1 and BRCA2 are the most frequently affected ones [6]. BRCA1 and BRCA2 are the tumor-suppressor genes located on chromosomes 17q21 and 13q12, respectively, that encode proteins playing vital roles in securing the stability of the DNA. Presence of inactivating variant in these genes may therefore result in improper repair of a DNA damage [7]. The frequency of pathogenic variants in both genes varies among populations worldwide, being the highest in the Ashkenazi Jewish population, in which they affect $2.5 \%$ of the general female population $[8,9]$. The result of genetic testing for the $B R C A$ variant is important for the management of breast cancer. Individuals with hereditary or familial breast and ovarian cancer are likely to benefit from strategies including prevention, screening and targeted treatment. Lack of mutational hot spots, presence of different types of variants, genomic rearrangements, and the sheer size of the genes are major challenges in the timely and efficient identification of mutant carriers.

Here we describe a rapid, multiplex and comprehensive screening approach for the detection of most frequent 
BRCA1 and BRCA2 variants occurring in Slovak HBOC population, which may prove to be valuable even in the era of NGS analysis. The BRCA1/2 screening test consists of two particular methods, SNaPshot analysis of selected variants in $B R C A 1 / 2$ genes and fragment analysis targeted for the insertions/deletions (ins/del) in exon 11 of BRCA1 gene. Currently, we are able to detect approximately $75 \%$ of all $B R C A 1 / 2$ causal variants reported in Slovak HBOC population, namely 13 variants in BRCA1 and 4 in BRCA2 gene.

\section{Patients and methods}

In this retrospective study, we have analyzed 687 DNA samples sent to the laboratory of Department of Clinical Genetics, St. Elizabeth Cancer Institute for BRCA1/2 screening test between 2012 and 2016. All analyzed individuals signed an informed consent and based on the family history, the samples were classified into two groups: those, who met minimally one of the below indication criteria (group 1, n=86) and those who did not (group 2, n=601).

Indication criteria:

- at least 2 patients with breast/ovarian cancer and 1 case before 50 years

- at least 3 patients of breast/ovarian cancer in one lineage

- $\quad$ one patient with breast/ovarian cancer and 1 case of associated carcinoma, 1 case before 50 years

- $\quad$ bilateral breast/ovarian cancer under 50 years

- sporadic breast/ovarian cancer under 40 years

- $\quad$ triple negative breast cancer under 50 years

- breast cancer in male patient.

Group 1 consists of individuals indicated for complex BRCA $1 / 2$ analysis and was comprised of 48 patients with diagnosed cancer disease and 38 healthy probands. The group of patients was composed of 38 breast cancer cases, 4 bilateral breast cancer cases, 2 ovarian cancer cases, 2 breast and ovarian cancer cases and 2 cases with other type of cancer.

Group 2 consists of individuals indicated only for the BRCA1/2 screening testing and was represented by 487 healthy individuals and 114 patients with diagnosed cancer but not fulfilled indication criteria. This set was composed of 90 breast cancer cases, 4 ovarian cancer cases, 8 cases with breast fibroadenomas and 12 cases with other type of cancer.

Genomic DNA was extracted from $2 \mathrm{ml}$ of peripheral blood using Genomic DNA Large Volume Whole Blood Kit (MagCore ${ }^{\circ}$. Multiplex PCR amplification was performed using Kapa2G Robust HotStart Mastermix (Kapa Biosystems), mix of 8 primer pairs and 100 ng of DNA template (Table S1). Samples were subsequently analyzed by SNaPshot analysis, which detected point variants in exon 2 (c.68_69del2), 5 (c.181T>G), 13 (c.4243delG), 20 (c.5266dupC) of BRCA1 and in exon 11 (c.3076A>T, c.5645C>A), 23 (c.9098dupA) and 25 (c.9403delC) of BRCA2 gene (Table 1). The primers for SNaPshot analysis were designed directly before or on the mutated nucleotide. SNaPshot analysis was performed using the SNaPshot Multiplex Kit (Thermo Fisher Scientific) according to the manufacturer's recommendations (Figure 1, part A). Fluorescent PCR amplification and fragment analysis was performed using Kapa2G Robust HotStart Mastermix (Kapa Biosystems), each primer and $60 \mathrm{ng}$ of DNA template. Fragment analysis was performed for the detection of the most relevant ins/del variants in three regions of exon 11 of $B R C A 1$ gene (Table 1). Migration of the fluorescent labeled fragments was compared to the size standard and the wild-type sample (Figure 1, part B).

Statistical analysis. All individuals' data were statistically described, analyzed for between-group differences, and summarized as means with respective standard deviations (SD) for continuous variables, or in contingency tables as counts or percentages. Agreement analysis was used to evaluate the degree of agreement and disagreement between both methods for the detection of variants in BRCA1/2 over categories in $2 \times 2$ tables. Diagnostic performance of the screening test was evaluated using the reference test (sequencing analysis). Cohen's kappa, Maxwell's chi-square and McNemar's statistics were used to test the agreement, disagreement and significant differences. The accuracy, sensitivity, specificity, positive predictive value (PPV), and negative predictive value (NPV) of the screening test were calculated. Diagnostic odds ratios (OR) for having the variant were estimated using the sample and the general population prevalence. All estimates are presented along with the respective $95 \%$ confidence intervals (95\% CI). Statistical analyzes were performed using StatsDirect 3.0.161 software (Stats Direct Ltd., Cheshire, UK). All reported p-values were two sided, and significance was set to $\mathrm{p}<0.05$.

Variants are reported in the context of a reference sequence, BRCA1 gene RefSeq NM_007294.3 and BRCA2 gene RefSeq NM_000059.3.

\section{Results}

In the screening test, we have used the combination of two detection methods. The SNaPshot analysis was used for the detection of specific variants in exons 2, 5, 13, 20 of BRCA1 and exons 11, 23, 25 of BRCA2. The fragment analysis was used for the detection of ins/del in exon 11 of BRCA1 gene (Table 1, Figure 1).

We have analyzed 687 patients, 86 of them (12.5\%) met the indication criteria for the complex analysis of $B R C A 1 / 2$ genes based on the positive family and personal history (group 1). We used the screening test to expedite the analysis and release of the results. The samples with negative results from screening were submitted for complex BRCA1/2 analysis. The other set of samples (group 2) consisted of 601 individuals $(87.5 \%)$ who did not meet the indication criteria for complex BRCA1/2 analysis, but the screening for the most frequent variants was recommended. Both groups of individuals were balanced with regard to age $(\mathrm{p}=0.77$; two-sample t-test). The overall average age was calculated at 52.23 years 
Table 1. Top: Characterization of the PCR products and SNaPshot primers. The mutation type, PCR product size, and type of wild-type (wt) and mutant (mut) nucleotide are indicated. Bottom: Characterization of the fragment analysis with mutations, fluorescent label, size and specified localization indicated.

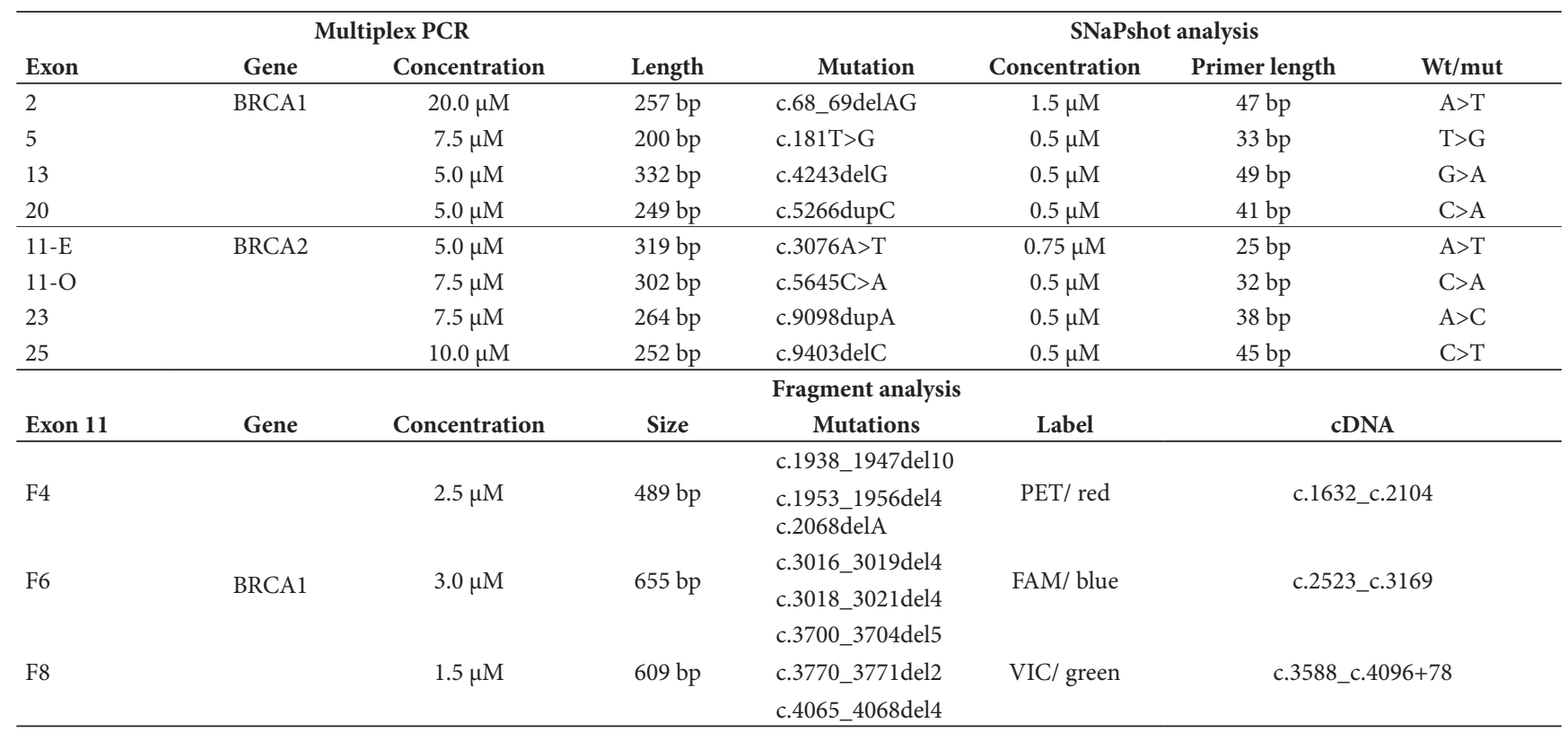

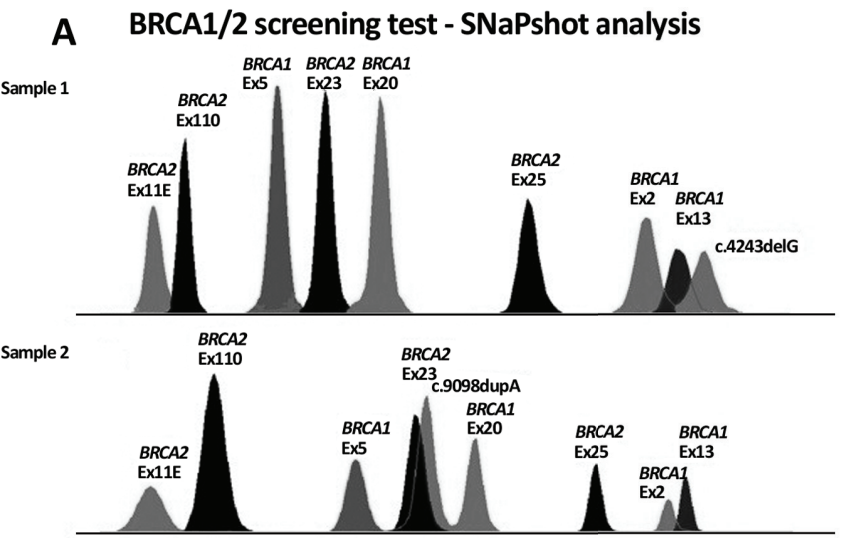

B BRCA1/2 screening test - Fragment analysis
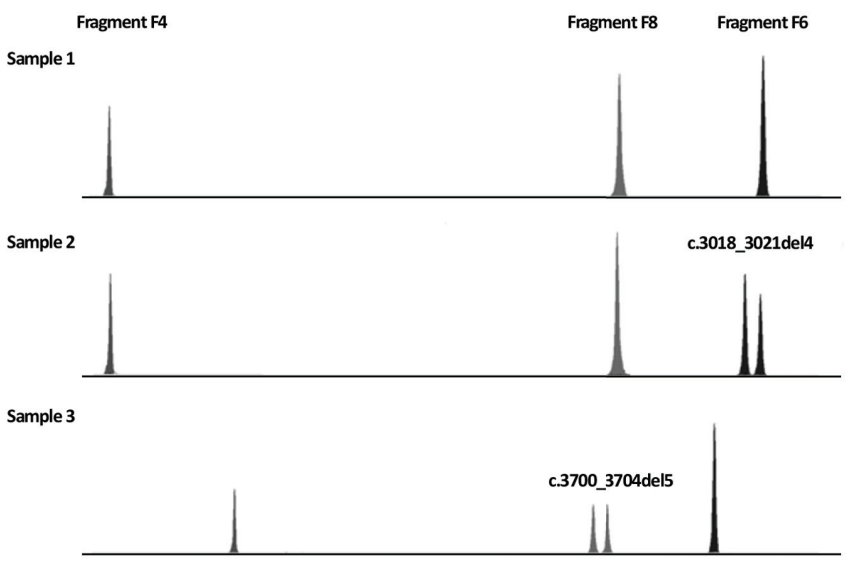

(range $20-87$ years), the average age of group 1 was calculated at 52.75 years (range 22-85) and group 2 average age at 50.75 (range 20-87). Presence of all detected variants was independently confirmed using the Sanger sequencing. This combined approach allowed us to identify 47 mutated samples (6.8\%), 40 in the group $1(46.5 \%)$ and 7 in the group 2 (1.1\%). Samples from group 1 were further submitted for complex analysis of $B R C A 1 / 2$ by massive parallel sequencing, which revealed 5 additional mutated samples (Table 2).

The subgroup of 7 positive samples of group 2 consisted of 6 healthy individuals and 1 patient with breast cancer diagnosed at the age of 55 and positive family history (breast cancer diagnosed in the sister aged 53). All 6 of the healthy positive individuals declared a positive family history, however, based on the Claus model scoring (using published tables) their estimated life-time risk for breast cancer was below the indication threshold 25\% (in the range 16-20\%).

All 45 positive individuals from group 1 were patients with diagnosed cancer: breast cancer in 33 , ovarian cancer in 3 , bilateral breast cancer in 3 , bilateral ovarian cancer in 1 , duplex of breast and ovarian cancer in 4 and duplex of

Figure 1. Examples of the results of SNaPshot and fragment analysis. A.) Selected results of SNaPshot multiplex analysis of BRCA1, BRCA2 genes. Sample 1 - Presence of deletion c.4243delG in exon 13 of BRCA1 gene is manifested by double peak A/G. Sample 2 - Presence of duplication c.9098dupA in exon 23 of $B R C A 2$ gene is manifested by double peak $A / G$. B.) Selected results of fluorescent multiplex PCR and fragment analysis of exon 11 of BRCA1 gene. Sample 1 - Sample without variant. Sample 2 - Presence of 4-base deletion. Sample 3 - Presence of 5-base deletion. 
Table 2. Variants detected using our diagnostic approach.

\begin{tabular}{|c|c|c|c|c|c|c|c|}
\hline c. DNA level & Protein level & Gene & Exon & Samples & Indicated & Not indicated & Method \\
\hline c. $181 \mathrm{~T}>\mathrm{G}$ & p.Cys61Gly & \multirow{8}{*}{$B R C A 1$} & 5 & 7 & 6 & 1 & SNP \\
\hline c.1881_1884del4 & p.Val627Serfs ${ }^{\star} 2$ & & \multirow{5}{*}{11} & 1 & 1 & 0 & FA \\
\hline c.1938_1947del10 & p.Ser646Argfs ${ }^{\star} 2$ & & & 1 & 1 & 0 & FA \\
\hline c.2068delA & p.Lys690Lysfs 10 & & & 2 & 2 & 0 & FA \\
\hline c.3018_3021del4 & p.His1006Glnfs ${ }^{*} 16$ & & & 8 & 7 & 1 & FA \\
\hline c.3700_3704del5 & p.Val1234Glnfs ${ }^{\star} 7$ & & & 3 & 1 & 2 & FA \\
\hline c.4243delG & p.Glu1415Lysfs ${ }^{\star} 3$ & & 13 & 2 & 2 & 0 & SNP \\
\hline c.5266dupC & p.Gln1756Profs ${ }^{\star} 73$ & & 20 & 15 & 13 & 2 & SNP \\
\hline c. $3076 \mathrm{~A}>\mathrm{T}$ & p. Lys $1026^{*}$ & \multirow{3}{*}{$B R C A 2$} & \multirow{2}{*}{11} & 3 & 2 & 1 & SNP \\
\hline c. $5645 \mathrm{C}>\mathrm{A}$ & p.Ser1882* & & & 1 & 1 & 0 & SNP \\
\hline c.9098dupA & p.Thr3033Asnfs ${ }^{*} 9$ & & 23 & 4 & 4 & 0 & SNP \\
\hline c. $4986+4 \mathrm{~A}>\mathrm{T}$ & exon 16 skipping & \multirow{2}{*}{$B R C A 1$} & 16 & 1 & 1 & 0 & MPS \\
\hline c. $5511 \mathrm{G}>\mathrm{A}$ & p.Trp $1837^{\star}$ & & 20 & 1 & 1 & 0 & MPS \\
\hline c. $4327 \mathrm{C}>\mathrm{T}$ & p.Arg1443* & \multirow{3}{*}{$B R C A 2$} & 13 & 1 & 1 & 0 & MPS \\
\hline c.7069_7070delCT & p.Leu2357Valfs ${ }^{\star} 1$ & & 14 & 1 & 1 & 0 & MPS \\
\hline c. $7558 \mathrm{C}>\mathrm{T}$ & p.Arg $2520^{*}$ & & 15 & 1 & 1 & 0 & MPS \\
\hline
\end{tabular}

SNP - SNaPshot analysis, FA - fluorescent PCR analysis, MPS - massive parallel sequencing.

bilateral breast and unilateral ovarian cancer in 1 case. The average age in this subgroup was 41.7 years $(\mathrm{SD}=10.11$, range 24-66).

Using the sequencing analysis for determining the variant status in group 1 allowed us to determine the extent to which the both readings would confirm. The proportion of correctly classified individuals was $88.9 \%(40 / 45)$ of positive and $100 \%$ $(86 / 86)$ of negative results. The negative and positive predictive values (NPV and PPV, respectively) were calculated using a prevalence of 52.3\% (95\%CI: 41.3-63.2\%) as found in the group 1 . The following results for the screening test were obtained:

- $\quad$ PPV was 100\% (95\%CI: 91.2-100\%),

- $\quad \mathrm{NPV}$ was $89.1 \%$ (95\%CI: 76.4-96.4\%),

- post-test variant likelihood despite negative test was 10.1\% (95\%CI: 3.6-23.6\%),

- $\quad$ sensitivity (true positive rate): $88.9 \%$ (95\%CI: 76.0-96.3\%),

- $\quad$ specificity (true negative rate): $100 \% \quad(95 \% \mathrm{CI}$ : 91.4-100\%).

To adapt the estimated PPV and NPV to the prevalence of $B R C A 1 / 2$ pathogenic variant in the general population, we used a value of $0.4 \%$ reported by Maxwell et al. (10) To recall, our "prevalence" based on the frequency of variant detected by the screening test and confirmed by sequencing in $100 \%$, amounted to $1.16 \%$. Assuming the detected perfect level of specificity and acceptable level for sensitivity of the screening test, we could easily show that recalculated PPV and NPV yielded $100 \%$ and $99.96 \%$, respectively.

Based on the results of the screening test which was conducted in both, group 1 and 2, the observed diagnostic odds ratio amounted to 72.6 (95\%CI: 30.1-203.1). Assuming the fact that sensitivity and specificity can be considered fixed properties of a diagnostic test, we could expect one person without indication classified as false negative. Thus, the odds ratio in our sample would change to 63.5 (95\%CI: 27.3-166.5), which means that a person with indication had on average 63.5 times higher odds of having variant than a person without indication.

\section{Discussion}

In our previous report, we have identified several variants in BRCA1/2 genes frequently occurring in Slovak population and therefore we have decided to design a rapid screening test. Altogether, nine variants (c.68_69delAG, c.181T $>$ G, c.3700_3704del5, c.4243delG, c.5266dupC in BRCA1, c.3076A > T, c.5645C >A, c.9098dupA, c.9403delC in BRCA2) accounted for $76 \%$ of the BRCA1/2-positive HBOC families (3). We designed our screening test as a combination of only two multiplex PCR reactions, variant-specific multiplex SNaPshot analysis and fluorescent multiplex PCR amplification followed by fragment analysis. Fragment analysis of possible ins/dels in exon 11 of BRCA 1 increased the theoretical detection limit of the test up to $80 \%$. Previous analysis of BRCA1/2 genes in Slovak HBOC families revealed variant detection frequency at the level of $17.1 \%$ [3]. The overall actual frequency of variants was only $6.8 \%$, which is in contrast with expected rate of $13.7 \%$ ( $80 \%$ of expected $17.1 \%$ variant carriers). However, we previously tested patients indicated for a complex BRCA1/2 analysis, while the current set included a considerable number of individuals who did not meet the criteria. Only $13 \%$ of tested samples came from patients indicated for the complex BRCA1/2 analysis (group 1). Interestingly, variant detection rate in this set was at the high level of $46.5 \%$. Brief comparison with other studies 
showed detection rate at $19.8 \%(n=197)$ [11] and $29.1 \%$ $(n=294)$ [12] in Czech, 27\% in Polish $(n=60)$ [13], 20\% in Austrian $(n=86)$ [14], $16 \%$ in Sicilian $(n=106)$ [15], and $17 \%$ in Spain population $(n=42)$ [16]. However, several factors, such as number of samples, composition of families, selection criteria or used screening techniques might contribute to this difference in the detection rate also between historically associated populations.

Similar screening approach was used by Filippini et al. [17] in Spanish HBOC population, however authors used only multiplex SNaPshot detection for ten recurrent and founder BRCA1/2 mutations, what represents approximately $50 \%$ of all observed Spanish mutations. All 48 samples were previously analyzed by Sanger sequencing and authors identified no false positive result. Another screening approach was published by Khachibi et al. [18] using HRM analysis for identification of mutations only in exon 11 of single BRCA1 gene, exactly in the group of 71 patients with diagnosed breast cancer. Altogether, they have identified causal mutations only in 2 samples with diagnosed breast cancer and positive family history. Cini et al. [19] published a study of the origin of most recurrent BRCA1/2 mutations in northeast Italy. For the quick identification of the eight frequent mutations they also developed a pre-screening test based on the in-house primer extension SNaPshot assay. Generally, using the rapid screening tests of BRCA1/2 mutations was previously mainly focused on the founder mutations specific for populations e.g. Ashkenazi Jewish [20], Iceland [21], Poland [22], Slovenia [23], Italy [24], North America [25] or Latin America [26] and were based as population specific genetic tests [27]. To our knowledge, this is the first publication which reports the rapid $B R C A 1 / 2$ screening test not targeted only at founder mutations and designed for such broad spectrum of BRCA1/2 mutations.

Further analysis of samples from group 1 by massive parallel sequencing identified 5 more pathogenic variants

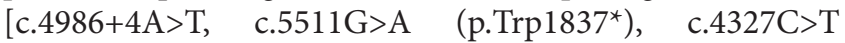
(p.Arg1443*), c.7069_7070delCT (p.Leu2357Valfs $\left.{ }^{\star} 1\right)$, c.7558C $\left.>\mathrm{T}\left(\mathrm{p} . \operatorname{Arg} 2520^{*}\right)\right]$. However, all were localized in parts of genes that were not included in the screening test, which strongly supports the high estimated specificity (100\%). Pathogenic variant carriers from group 1 were all diagnosed with some type of cancer, majority of carriers were diagnosed with breast cancer $(73 \%)$, duplex of breast and ovarian cancer was diagnosed in $8 \%$, ovarian cancer or bilateral breast cancer in $6.7 \%$.

As mentioned before, for patients from group 2 the test for most frequent variants was recommended for various reasons, e.g. psychological indications, personal decision to undergo the test, positive family history or positive personal history below the threshold criteria. In this group of 601 samples, we have detected the presence of pathogenic variants only in $1.1 \%$ of samples. The common characteristic of these samples was positive family history, which appears to be the strongest criterion for the DNA analysis.
The most frequently detected variant in the analyzed group was c.5266dupC identified in 32\%, followed by variant c.3018_3021del5 detected in $17 \%$ and exon 5 variant c. $181 \mathrm{~T}>\mathrm{G}$ detected in $15 \%$ of $B R C A 1$ variant positive families. These three variants were also most frequent ones in previous reports $(3,12)$. The most frequently detected $B R C A 2$ variant was c. $5645 \mathrm{C}>\mathrm{A}$ identified in $8.5 \%$ of positive families. As expected also in group 2, the most frequent variant c.5266dupC in BRCA1 was identified in 2 individuals. Surprisingly, a relatively rare variant c.3700_3704del5 in $B R C A 1$ gene was also detected in two individuals. Other three variants (c.181T $>\mathrm{G}$, c.3018_3021del4 in BRCA1 and c.3076A $>\mathrm{T}$ in BRCA2) were determined each in one individual. It needs to be stated that based on the recent data about the prevalence of BRCA2 variants in central European region, we need to widen the spectrum of variants.

Using statistical analysis, the observed odds ratio of the screening BRCA1/2 test was estimated to 73.8 and the conditional maximum likelihood estimate was 72.6 (30.1 to 203.1). Sensitivity (true positive rate) of this approach was counted at $89 \%$ (range $75.9 \%$ to $96.3 \%$ ) and specificity (true negative rate) was determined to $100 \%$ (range $91.4 \%$ to $100 \%$ ).

We take into account the general population prevalence of $B R C A 1 / 2$ pathogenic mutation $(0.4 \%)$ and detected levels of specificity and sensitivity to define positive and negative predictive value of the screening test. Lower positive predictive values of the test (4\%) show that we can expect some results as false positive, however these samples should undergo approbation by Sanger sequencing. On the other side, negative predictive value of the screening test reaches $99.9 \%$.

With rare conditions, the PPV is driven by specificity. If it were the specificity different from $100 \%$, for example, if we use the lower 95\% confidence limit for the specificity, the falling prevalence would lead to a rapid decrease in PPV (4\%) and thus, in an increase in the false positive results. This should be kept in mind when estimating the real-life performance of the screening method, and positive samples should always undergo approbation by Sanger sequencing.

In the conclusion, we suppose that even in the bioinformatic NGS era this simple, rapid, and cost-effective test represents a reliable alternative, sufficiently sensitive to identify individuals in the targeted part of population who have an increased lifetime risk for developing cancer, but do not meet the standard selection criteria. Naturally, the test would not provide complex results, but it offers testing accessible to a broader spectrum of individuals, whose clinical history puts them just under the threshold of indication for complex BRCA1/2 analysis.

Acknowledgements: This publication is a result of the project implementation: 'Establishment of competence Centre for research and development in the field of molecular medicine'. Supported by the Research \& Development Operational Program funded by the ERDF (ITMS 26240220071). We would like to thank Jan Markus, $\mathrm{Ph} . \mathrm{D}$. for editorial assistance in the preparation of the manuscript. 


\section{References}

[1] FERLAY J, SOERJOMATARAM I, DIKSHIT R, ESER S, MATHERS $\mathrm{C}$ et al. Cancer incidence and mortality worldwide: sources, methods and major patterns in GLOBOCAN 2012. Int J Cancer 2015; 136: E359-386. doi: 10.1002/ ijc. 29210

[2] FERLAY J, STELIAROVA-FOUCHER E, LORTET-TIEULENT J, RUSSO S, COEBERGH JW et al. Cancer incidence and mortality patterns in Europe: estimates for 40 countries in 2012. Eur J Cancer 2013; 49: 1374-1403. doi: 10.1016/j. ejca.2012.12.027

[3] KONECNY M, MILLY M, ZAVODNA K, WEISMANOVA E, GREGOROVA J et al. Comprehensive genetic characterization of hereditary breast/ovarian cancer families from Slovakia. Breast Cancer Res Treat 2011; 126: 119-130. doi: 10.1007/s10549-010-1325-x

[4] COLLABORATIVE GROUP ON HORMONAL FACTORS IN BREAST CANCER. Familial breast cancer: collaborative reanalysis of individual data from 52 epidemiological studies including 58,209 women with breast cancer and 101,986 women without the disease. Lancet 2001; 358: 1389-1399. doi: 10.1016/S0140-6736(01)06524-2

[5] CAMPEAU PM, FOULKES WD, TISCHKOWITZ MD. Hereditary breast cancer: new genetic developments, new therapeutic avenues. Hum Genet 2008; 124: 31-42. doi: 10.1007/ s00439-008-0529-1

[6] RAHMAN N. Realizing the promise of cancer predisposition genes. Nature 2014; 505: 302-308. doi: 10.1038/nature12981

[7] GUDMUNDSDOTTIR K, ASHWORTH A. The roles of BRCA1 and BRCA2 and associated proteins in the maintenance of genomic stability. Oncogene 2006; 25: 5864-5874. doi: 10.1038/sj.onc.1209874

[8] KLEIBL Z, KRISTENSEN VN. Women at high risk of breast cancer: Molecular characteristics, clinical presentation and management. Breast 2016; 28: 136-144. doi: 10.1016/j. breast.2016.05.006

[9] WARNER E, FOULKES W, GOODWIN P, MESCHINO W, BLONDAL $J$ et al. Prevalence and penetrance of BRCA1 and BRCA2 gene mutations in unselected Ashkenazi Jewish women with breast cancer. J Natl Cancer Inst 1999; 91: 1241-1247.

[10] MAXWELL KM, DOMCHEK SN, NATHANSON KL, ROBSON ME. Population frequency of germline BRCA1/2 mutations. J Clin Oncol 2016; 34: 4183-4185. doi: 10.1200/ JCO.2016.67.0554

[11] FORETOVA L, MACHACKOVA E, NAVRATILOVA M, PAVLU H, HRUBA $M$ et al. BRCA1 and BRCA2 mutations in women with familial or early-onset breast/ovarian cancer in the Czech Republic. Hum Mutat 2004; 23: 397-398. doi: 10.1002/humu.9226

[12] MACHACKOVA E, FORETOVA L, LUKESOVA M, VASICKOVA P, NAVRATILOVA $M$ et al. Spectrum and characterisation of BRCA1 and BRCA2 deleterious mutations in high-risk Czech patients with breast and/or ovarian cancer. BMC Cancer 2008; 8: 140. doi: 10.1186/1471-2407-8-140
[13] PERKOWSKA M, BROZEK I, WYSOCKA B, HARALDSSON K, SANDBERG T et al. BRCA1 and BRCA2 mutation analysis in breast ovarian cancer families from northeastern Poland. Hum Mutat 2003; 21: 553-554. doi: 10.1002/ humu.9139

[14] WAGNER TM, MOSLINGER RA, MUHR D, LANGBAUER G, HIRTENLEHNER $\mathrm{K}$ et al. BRCA1-related breast cancer in Austrian breast and ovarian cancer families: specific BRCA1 mutations and pathological characteristics. Int J Cancer 1998; 77: 354-360.

[15] RUSSO A, CALÒ V, AGNESE V, BRUINO L, CORSALE S et al. BRCA1 genetic testing in 106 breast and ovarian cancer families from Southern Italy (Sicily): a mutation analyzes. Breast Cancer Res Treat 2007; 105: 267-276. doi: 10.1007/ s10549-006-9456-9

[16] LLORT G, MUNOZ CY, TUSER MP, GULLERMO IB, LLUCH JR et al. Low frequency of recurrent BRCA1 and BRCA2 mutations in Spain. Hum Mutat 2002; 19: 307. doi: 10.1002/humu.9014

[17] FILIPPINI S, BLANCO A, FERNÁNDEZ-MARMIESSE A, ÁLVAREZ-IGLESIAS V, RUÍZ-PONTE C et al. Multiplex SNaPshot for detection of BRCA1/2 common mutations in Spanish and Spanish related breast/ovarian cancer families. BMC Med Genet 2007; 8: 40. doi: 10.1186/1471-2350-8-40

[18] EL KHACHIBI M, DIAKITE B, HAMZI K, BADOU A, SENHAJI MA et al. Screening of exon 11 of BRCA1 gene using the high-resolution melting approach for diagnosis in Moroccan breast cancer patients. BMC Cancer 2015; 15: 81. doi: 10.1186/s12885-015-1040-4

[19] CINI G, MEZZAVILLA M, DELLA PUPPA L, CUPELLI E, FORNASIN A et al. Tracking of the origin of recurrent mutations of the BRCA1 and BRCA2 genes in the North-East of Italy and improved mutation analysis strategy. BMC Med Genet 2016; 17: 11. doi: 10.1186/s12881-016-0274-6

[20] LEVY-LAHAD E, CATANE R, EISENBERG S, KAUFMAN B, HORNREICH G et al. Founder BRCA1 and BRCA2 mutations in Ashkenazi Jews in Israel: frequency and differential penetrance in ovarian cancer and in breast-ovarian cancer families. Am J Hum Genet 1997; 60: 1059-1067.

[21] THORLACIUS S, SIGURDSSON S, BJARNADOTTIR H, OLAFSDOTTIR G, JONASSON JG et al. Study of a single BRCA2 mutation with high carrier frequency in a small population. Am J Hum Genet 1997; 60: 1079-1084.

[22] GÓRSKI B, JAKUBOWSKA A, HUZARSKI T, BYRSKI T, GRONWALD J et al. A high proportion of founder BRCA1 mutations in Polish breast cancer families. Int J Cancer 2004; 110: 683-686. doi: 10.1002/ijc.20162

[23] KRAJC M, TEUGELS E, ZGAJNAR J, GOELEN G, BESIC $\mathrm{N}$ et al. Five recurrent BRCA1/2 mutations are responsible for cancer predisposition in the majority of Slovenian breast cancer families. BMC Med Genet 2008; 9: 83. doi: 10.1186/1471-2350-9-83

[24] CALECA L, PUTIGNANO AL, COLOMBO M, CONGREGATI C, SARKAR $M$ et al. Characterization of an Italian founder mutation in the RING-finger domain of BRCA1. PLoS One 2014; 9: e86924. doi: 10.1371/journal. pone. 0086924 
[25] FERLA R, CALÒ V, CASCIO S, RINALDI G, BADALAMENTI $G$ et al. Founder mutations in BRCA1 and BRCA2 genes. Ann Oncol 2007; Suppl 6: vi93-98. doi: 10.1093/annonc/mdm 234

[26] OSSA CA, TORRES D. Founder and Recurrent Mutations in BRCA1 and BRCA2 Genes in Latin American Countries: State of the Art and Literature Review. Oncologist 2016; 21 : 832-839. doi: 10.1634/theoncologist.2015-0416
[27] FOULKES WD, KNOPPERS BM, TURNBUlL C. Population genetic testing for cancer susceptibility: founder mutations to genomes. Nat Rev Clin Oncol 2016; 13: 41-54. doi: $10.1038 /$ nrclinonc. 2015.173 